\title{
Integrated modeling of a complex oil rim development scenario under subsurface uncertainty
}

\author{
Mansour Elharith ${ }^{1} \cdot$ Ho Yeek Huey $^{1} \cdot$ Raj Deo Tewari $^{1} \cdot$ Lisa Claire $^{1} \cdot$ Nurul Suhaila M. Fawzi $^{2} \cdot$ Ralf Schulze-Riegert $^{3}$
}

Received: 29 November 2018 / Accepted: 5 March 2019 / Published online: 16 March 2019

(c) The Author(s) 2019

\begin{abstract}
Evaluation of the economic benefit of field development projects in the oil and gas industry is naturally subject to subsurface uncertainties and risks. While high economic returns from produced volumes compensated for the lack of robust uncertainty estimates in the past, lower margins in tight market conditions and more complex project designs require increased efforts in delivering reliable uncertainty estimates for improving reservoir management decision support in times to come. An increasing number of field development projects include rigorous uncertainty quantification workflows based on parameterized subsurface uncertainties. Estimation and reduction of prediction uncertainty has no value unless it can potentially influence a decision process. This requires an integrated workflow design across disciplines and a proper risk mitigation plan. This work presents the implementation of an uncertainty workflow in reservoir management with a focus on reservoir modeling and simulation. Practical workflow design steps and intermediate milestones are discussed for generating alternative reservoir model realizations including historical production data. Calibrated model realizations define the basis for prediction estimates of a predefined field development plan. Practical steps are discussed with application to a complex oil rim development for an existing reservoir with long production history. This study aimed at an uncertainty assessment of a field development plan and a proposal for optimized relocations of infill wells considering multiple geological realizations. In conclusion, this work describes examples, experiences, and recommendations for an industry implementation for a risk assessment of a field development plan under subsurface uncertainties.
\end{abstract}

Keywords Uncertainty quantification $\cdot$ History matching $\cdot$ Estimation prediction uncertainty $\cdot$ Integrated modeling $\cdot$ Field development planning under uncertainty $\cdot$ Reservoir management

Mansour Elharith

m_mansour@petronas.com

Ho Yeek Huey

hoyeekhuey@petronas.com

Raj Deo Tewari

rajtewari@petronas.com

Lisa Claire

lisaclaire.chisholm@petronas.com

Nurul Suhaila M. Fawzi

NFawzi@slb.com

Ralf Schulze-Riegert

RSchulze-Riegert@slb.com

Petronas, Kuala Lumpur, Malaysia

2 Schlumberger, Kuala Lumpur 50450, Malaysia

3 Schlumberger Norwegian Technology Center, Asker 1383, Norway

\section{Introduction}

Oil rim development scenarios are subject to significant risks due to subsurface uncertainties with a strong impact on the economics of a field development plan. A full uncertainty study can support or decline a field development plan if the robustness of an economic benefit cannot be proven for all probable subsurface realizations (Komin 2018).

Incorporation of dynamic field data brings reliability to static description/characterization of reservoirs and fields. The uncertainty level is high in green fields which has no production history. The predictability of reservoir models of brown fields is not free of uncertainty and depends on the maturity of the data integration process to calibrate models. Generally, uncertainty reduces with time, but deviations are observed in actual production behaviors with respect to forecasts in a majority of fields. This challenge is not specific to 
a particular company but to the industry as a whole (Nandurdikar and Wallace 2011).

An exact solution to a reservoir engineering problem does not exist due to the large size of reservoirs, complex nature of fluid distribution in the porous media, and the number of influencing variables. Therefore, approximations are implemented to work with manageable solutions.

\section{Practical approach for managing subsurface uncertainties and risks}

Scenario-based cases are generated to understand the minimum and maximum outcomes along with average values. This process delivers an estimate of ranges in outcomes; however, results may be far from being realistic. All minimum parameter values in one case for the low outcome and similarly all the maximum parameter values for the high outcome may not be a good representation of reality. Outcomes are often over- or underestimated. The choice of deterministic models does not sufficiently address subsurface uncertainties. Proper risk management should look at a probabilistic formulation of understanding the reservoir description and modeling of fluid flow in porous media.

Brute force probabilistic modeling requires a large number of simulation runs and is not feasible for most practical reservoir studies. It becomes important to understand the impact of model parameter variations on outcomes. Fluid flow in the porous medium is a complex phenomenon and a large number of parameters are influential. All these parameters carry various degrees of uncertainty and cannot be given equal treatment as they will increase computing requirements drastically and delay the completion of a study. Therefore, parameters with a significant impact must be identified and prioritized in the risk assessment.

In this work we adopt a two-step approach. Uncertainties in static modeling are first investigated with respect to volumetric and connectivity-related performance indicators. In a second step, relevant static and dynamic uncertainty parameters are included in a model validation process, called history matching. A number of trusted candidate solutions are moved forward to prediction to assess uncertainty ranges for future production for a base case field development scenario. Probability maps (Fig. 1) are used to visualize a mutual view on all alternative realizations to identify high-probability oil saturation regions at the start of the production forecast for deciding on optimized infill drilling locations.

The paper is organized in three main sections: methodology introduction, workflow design, and application.

\section{Workflow design, modeling phases, and methodology}

A complete workflow design for history matching and selection of prediction candidates built on standard experimental design techniques coupled with several data analysis methods is described in Bhark and Dehghani (2014). An ensemble-based approach for history matching and uncertainty quantification is described in Hanea et al. (2015) to support "fast model updates" as part of a closed-loop workflow design. A common part among these history matching

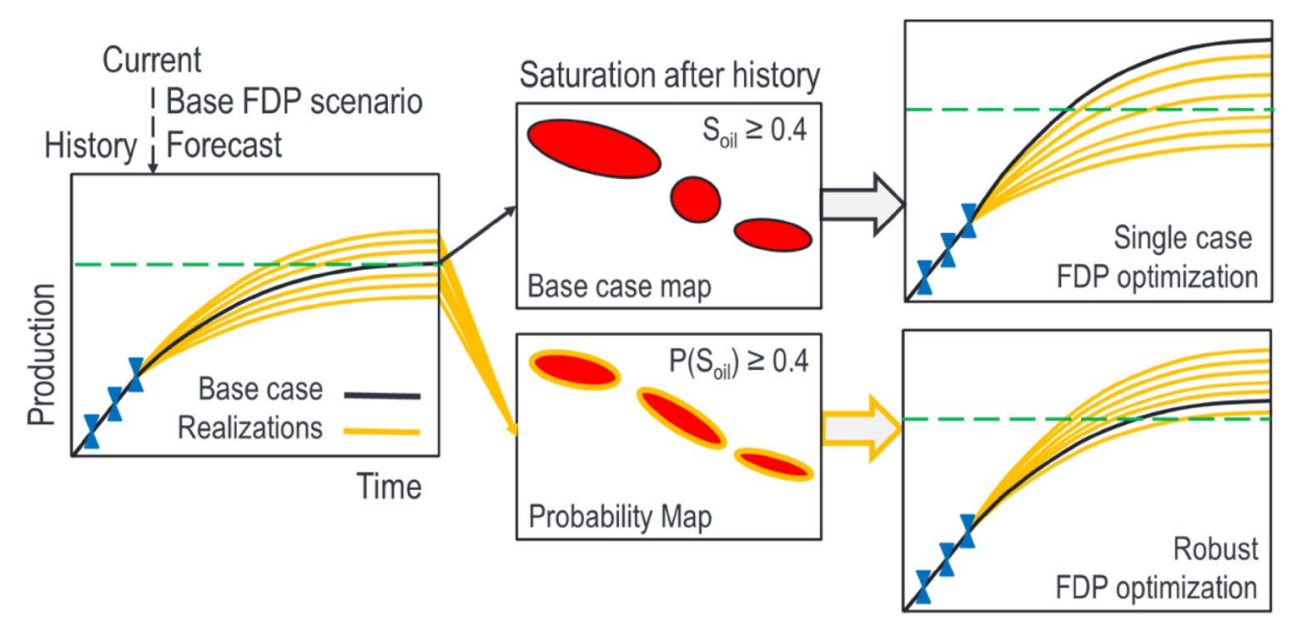

Fig. 1 Multiple-realizations are history matched and meet historical production data (left). Multiple realizations (orange lines) are compared to a base case model (black line) for a field development scenario. At the current point of production, the area with remaining oil saturation higher than $40 \%$ is visualized for the base case scenario (upper middle). For the same time point of production a probability map visualizes areas with a probability finding oil saturation higher than $40 \%$ based on all available multiple realizations (lower middle). An optimized FDP based on a single case shows a broad distribution of outcomes (upper right). An optimized FDP based on multiple realizations (lower right) is more robust and shows a narrow distribution of outcomes which represent an overall lower risk potential 
Fig. 2 Generic workflow for risk assessment of a field development plant (FDP)

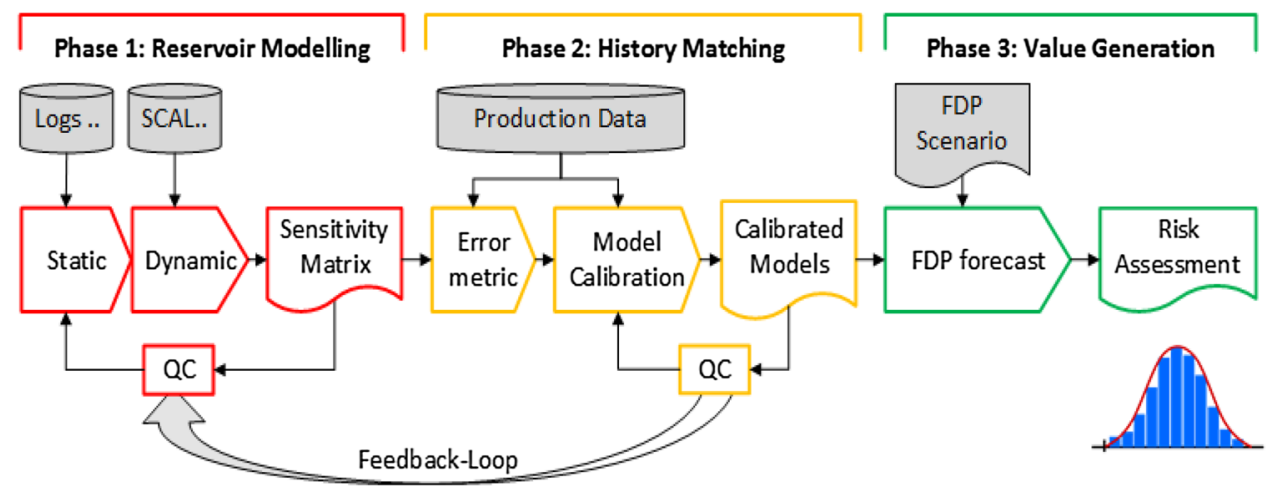

workflows is that they are based on integrated multi-disciplinary team work to support model consistency across multiple modeling phases.

In this section we describe a generalized framework for probabilistic forecasting including historical production data. An extended description and related works is included in Schulze-Riegert et al. (2017a, b). Figure 2 captures basic modeling phases for risk assessment of a field development plan based on subsurface uncertainties. Reservoir modeling (1) supported by basic sensitivity studies prepares the input and framing for the model calibration phase (2). An error metric defines an objective measure to quantify the mismatch between historical and simulated measurement data used in assisted history matching. The actual value is created in phase (3) using multiple calibrated models for uncertainty and risk assessment of field development scenarios.

\section{Reservoir modeling}

Uncertainty assessment of the static modeling workflow uses Monte-Carlo sampling to estimate a distribution of oil-inplace. Examples for input domains to the static reservoir modeling are shown in Fig. 3.

Due to the nature of the complexity of the modeling aspects, discrete scenarios are used for selected domains and continuous distributions for parameters where it is applicable. Ranges for each parameter are motivated from existing well control or analogue field data.

Sensitivity studies are used to investigate the impact of parameter domains. An example for the result analysis of the geological modeling process is shown in Fig. 4. The tornado plot shows the relative impact of high and low parameter values with respect to a reference case. Not all scenarios can be moved into dynamic modeling and representative cases need to be selected. For this purpose multi-dimensional ranking techniques are used in connection with fast flow-based approximation models which are capable to assess connected volumes as an indicator for production performance.

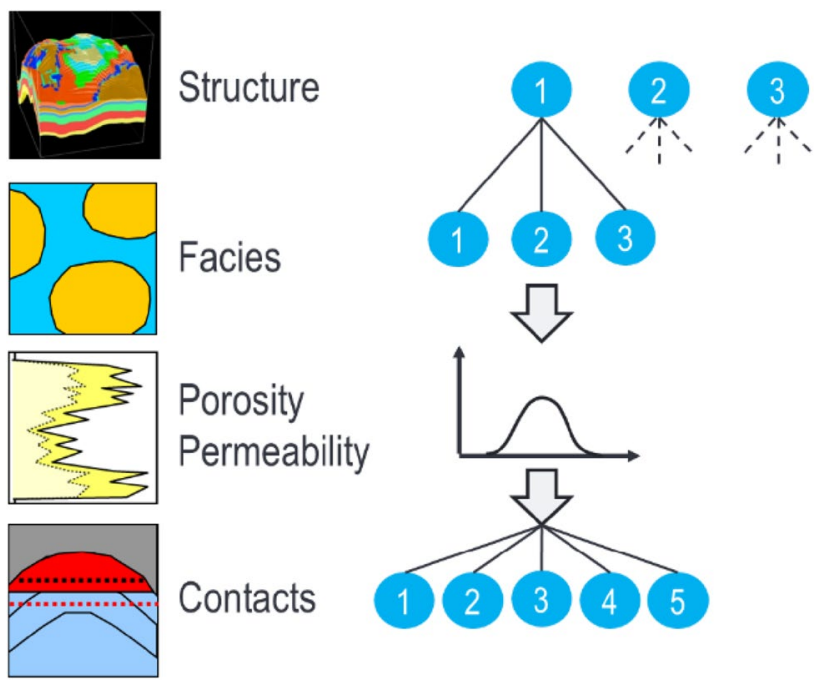

Fig. 3 Hierarchy of input parameters and resultant volumetric cases created

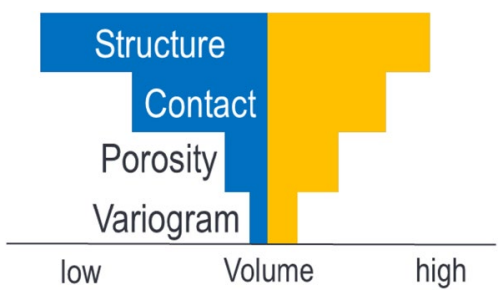

Fig. 4 Tornado diagram showing the impact on uncertainty parameters on reservoir in place volumes

\section{History matching using Markov chain Monte Carlo (MCMC)}

MCMC sampling algorithms are used to explore the full posterior probability density function which puts a large weight on cases with a small mismatch and a small weight on cases with a large mismatch between simulated and historical measurment data (Slotte and Smorgrav 2008; 
Schulze-Riegert et al. 2016, 2017a, b). This process involves a large number of function evaluations which would be unfeasible using full field simulations.

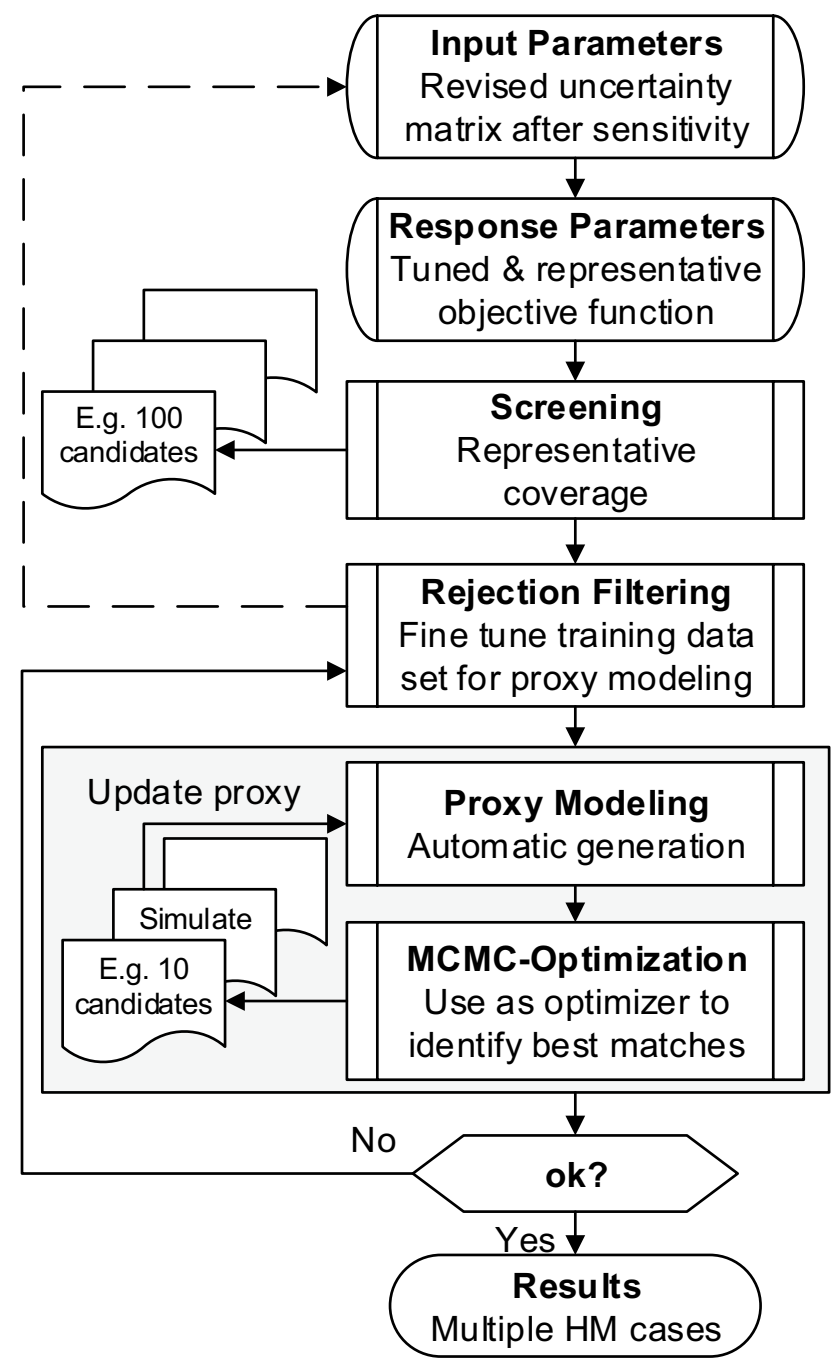

Fig. 5 History Matching supported by MCMC techniques
For that purpose, proxy models are built to represent the posterior probability density function. The number of response parameters included in the posterior probability density function can be large and automatic proxy modeling techniques become mandatory. In an iterative refinement process Fig. 5, proxy models become a continuously improved representation of the posterior distribution function for sampling history matched solution candidates (Schulze-Riegert et al. 2017a, b).

The history matching workflow design Fig. 5 is depending on a number of definition and quality control steps which have a significant influence on the performance of the sampling process of history matched candidate solutions. Input parameter definitions are revised after a full sensitivity study which includes ranges as well as the deactivation of parameters in case the impact on history matching objectives is shown to be small. Response parameters need to be a reliable representation of the history matching objective. This typically includes a tuning process to guarantee that the global objective function value converges to a target solution which includes the sum of all mismatch contributions.

This type of global objective quality criteria is often problematic since it does not allow to distinguish individual contributions, Fig. 6 . The left plot shows optimization results sorted according to the global objective value. For the given example, lowest global objective function values do not necessarily correlate with small pressure or small rate mismatch values. The same observation is given on the cross plot (right side). A low rate-mismatch could be linked to a large pressure-mismatch and vice versa. This underlines the multi-objective nature of many history matching studies. This observation is often an indication that the uncertainty parameterization (static and dynamic model) is not complete.

Rejection filtering is used to address this observation. Rejection filtering defines a multi-level selection scheme and is useful in various phases of the workflow. The benefit of rejection filtering is the ability to focus on multi-objective
Fig. 6 Multi-objective criteria shown as staged-bar-plot (left) and cross-plot (right)

\section{Global Mismatch Contributions}

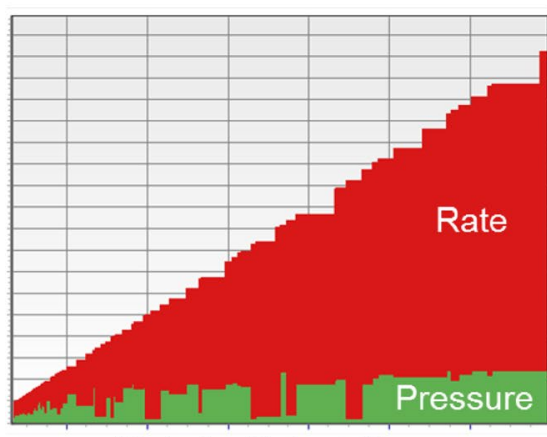

Global $=$ Rate + Pressure

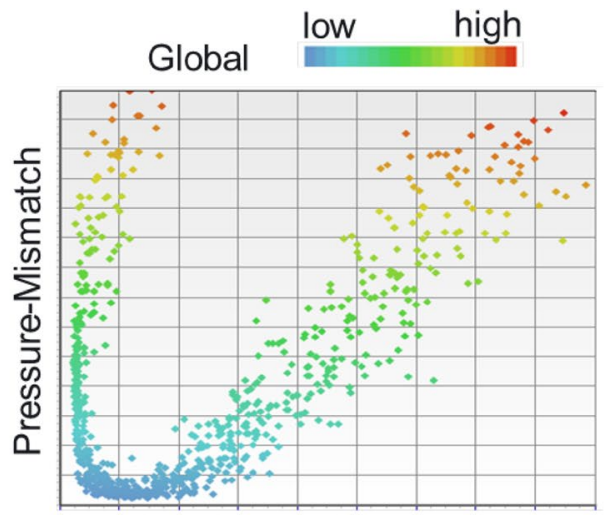

Rate-Mismatch 
criteria separately, e.g., pressure match vs. rate match shown in Fig. 6. Extreme cases can be easily identified and excluded from the training data set which is used in the automatic proxy generation process.

History matching solutions are typically found after a few iterations with less full field simulation runs compared to the original screening data set. This makes this workflow design efficient also for complex simulation cases.

\section{Probability maps for well location optimization}

Uncertainty analysis for reservoir performance usually involves running hundreds of reservoir simulations. The outputs of the reservoir simulator may include fluid saturations, compositions, pressures, temperature, and other physical properties of interest at every grid block of the reservoir model. Furthermore, outputs may include wellbore related to performance such as water-cut, gas-cut, phase transitions, and total flow-rate. It is not uncommon for a typical reservoir to be represented by hundred thousand to several millions of grid cells. As a result, a vast amount of data are generated. In the absence of efficient visualization and analysis tools, only a small subset of the generated data is used for subsequent decision-making. Conveying vast data and its uncertainty is particularly important for enabling informed decisions with regard to acquire future data so as to reduce future uncertainties.

One way to do this is by generating probability maps (Chugunov 2013, 2015), where each grid cell of the reservoir is represented by a probability of a given physical property to exceed a predefined threshold value. As discussed in Fig. 1, such representations prove to be useful in evaluating spatial probabilistic performance of the reservoir over time and become instrumental in analysis ensembles reservoir realizations (Schulze-Riegert et al. 2017a, b; Komin 2018).

The concept for calculating probability maps is schematically shown in Fig. 7. Property distributions are calculated for every grid cell over an ensemble of 100 models. A probability map in this example would show the probability finding saturation oil larger than the predefined threshold value of $70 \%$.

In this work probability maps will be used to assess development areas of interest for new infill wells under uncertainty. Maps will be calculated based on multiple history matched models which are selected for predication.

\section{Application}

This section gives a detailed overview of the application and presents the results and lesson learned.

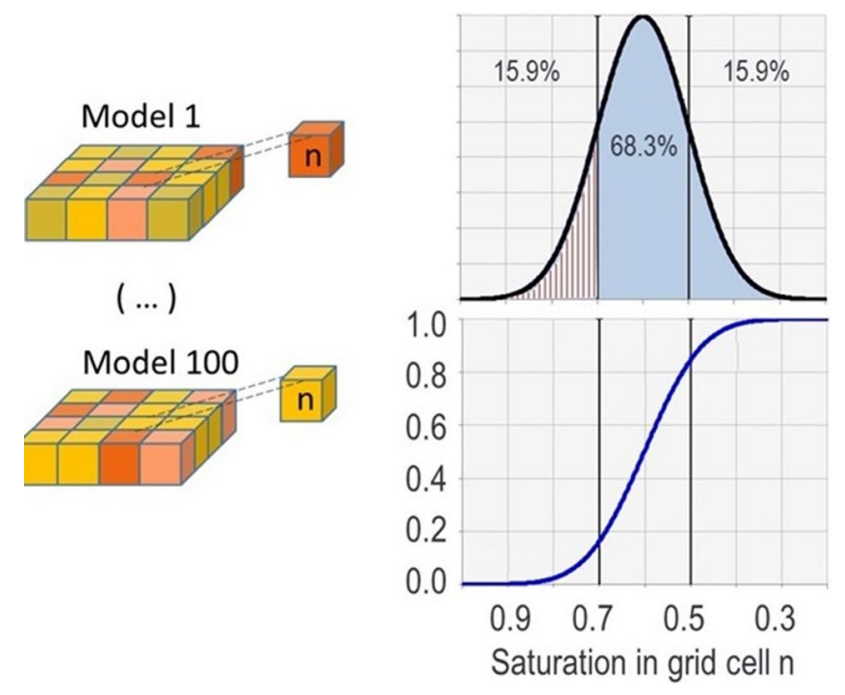

Fig. 7 Concept for calculating probability maps

\section{Scope of the project}

The objective of this study is to understand key uncertainties that impact the performance of an oil-rim development strategy of XX-XY reservoir. The uncertainty assessment of a baseline development strategy is built on multiple history matched models following the workflow design described in the previous section.

The baseline development strategy includes drilling targets of three water injectors, one gas injector and three oil producers to further improve the reservoir recovery factor. The development is planned in two phases. Water and gas injectors will be drilled in a first phase followed by oil producers that will be drilled in a second phase. This will allow to confirm the current oil rim thickness and to improve the location of oil producers.

Understanding oil rim movement is critical for optimizing the field development plan. This includes water and gas injection strategy, infill well locations, as well as optimum perforation intervals for workover candidates. The uncertainties affecting oil rim position and thickness are expected to be gas-oil and water-oil contacts (defines thickness of initial oil column), vertical connectivity, lateral connectivity, relative aquifer strengths between eastern and western flanks (oil rim tilting), gas cap expansion, and historical gas injection. Other production uncertainties include gas leak to other reservoirs through behind casing channeling, in loop gas injection from deep reservoirs as well as production metering allocation.

Another uncertainty aspect affecting oil recovery from this reservoir is the amount of residual oil left behind after introducing water flooding. The available water flooding

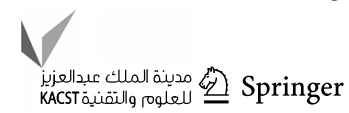


core experiments show that residual oil saturation varies between 10 and $30 \%$ pore volume.

This paper will focus on the uncertainty assessment for a baseline field development scenario. Probability maps for movable oil will be used to verify the plausibility of baseline infill drilling locations.

\section{Reservoir model description}

The reservoir described in this work is operated under the influence of a large initial gas cap and a down dip aquifer support. This work gives a focus on the oil-rim reservoir which has produced from 46 wells over 44 years. The reservoir is a Miocene shallow-marine sandstone, which shows repeated progradation and retrogradation within a major regressive clastic wedge which prograde towards the NW. The reservoir comprises two major units, $\mathrm{XX}$ and $\mathrm{XY}$. Although the two units share a common Gas-Oil Contact (GOC), they are geologically distinct units separated by laterally extensive intra-reservoir shale with a thickness increasing westward from $6 \mathrm{ft}$ to $16 \mathrm{ft}$ as shown in Fig. 8 . The non-uniform pressure depletion within these two units suggested that the shale layer is sealing or partially sealing. Communication through this shale could be due to thickness variations, occasional interbedded sand layers, and an offset near minor sub-seismic faults related to major north and south faults.

Geologically, the sands are interpreted to be continuous bodies forming the upper to middle shore face deposits. The porosity ranges from 20 to $35 \%$ and permeability ranges between 50 milli-Darcy to 2 Darcy. The geological column
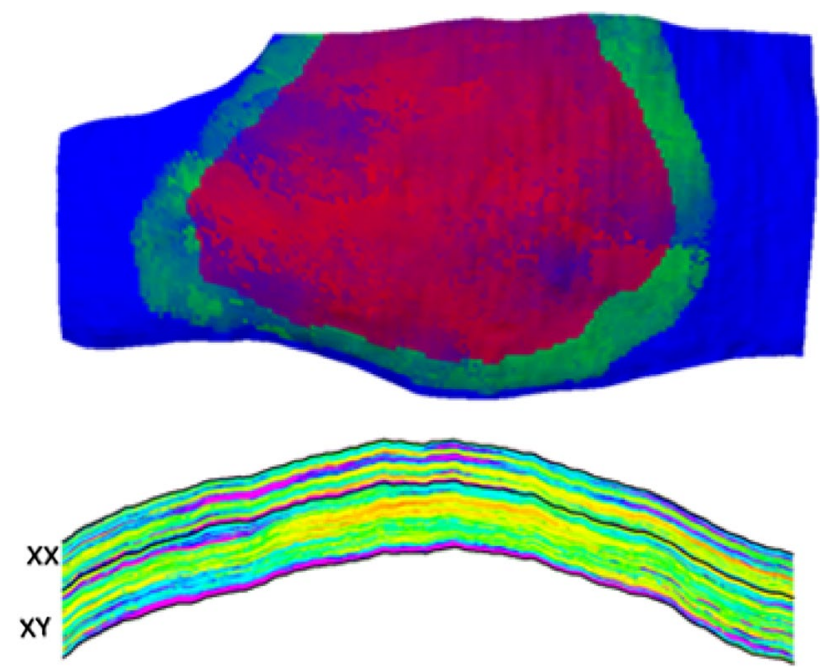

Fig. 8 Top view of 3-phase saturation distribution (top), gas (red), oil (green) saturation. East-west cross-section of permeability distribution (bottom) in the field mainly consists of clastic deposits characterized by thick sands inter-bedded with thin shale layers.

The oil rim has an average thickness of $106 \mathrm{ft}$ throughout the field, overlaid by $\sim 300 \mathrm{ft}$ of a gas cap mainly occupying the top reservoir. The large gas cap has effectively supported reservoir pressure and delayed pressure depletion. Due to increasing production from the reservoir, gas injection was introduced in 1994 from two crestal wells to maintain reservoir pressure and to prevent desaturation of oil to gas cap. Although material balance does not indicate a very strong aquifer support, the high water cut observed in most of the wells indicates some aquifer activities.

A base case static model with 2.4 million cells was selected for constructing the dynamic model. Prior to the history matching stage the dynamic model was upscaled to 1.4 million cells which helped to reduce the run time by $40 \%$. Important reservoir features (thin shale layers, variogram size) were preserved and upscaled model results were properly quality checked vs. the static model results. Furthermore, high end computing infrastructure was used along with high-resolution simulation software to further reduce the run time.

\section{Reservoir uncertainties and challenges}

A series of discussions between various disciplines within the team helped to outline the major surface/subsurface uncertainties that could have an impact on reservoir performance and on the history matching quality.

Figure 9 illustrates key uncertainty sources identified for the $\mathrm{XX}-\mathrm{XY}$ reservoirs.

From a subsurface point of view both static and dynamic uncertainties have been identified. A reasonable range of parameter values was developed for each parameter based on available measurement data and observations on analogues reservoirs.

Due to the high density of wells in the reservoir, no significant difference on variogram size was expected, hence a base case static model variogram was selected and an uncertainty range was defined around it. This allowed to parameterize static uncertainties as continuous parameters which simplified the optimization step in the history matching workflow. Property distributions in the flanks (aquifers), where less wells were drilled, were captured by transmissibility regions. Vertical transmissibility between layers was incorporated to understand interlayer connectivity.

Petrophysical evaluations performed on cores and well logs indicate that the estimation of initial gas-oil contact and Free Water Level (FWL) are subjected to \pm 10 feet variation around the most likely case contacts. The reliability of low and high case contacts has been verified by cross checking 
Fig. 9 Key uncertainties in XX$X Y$ reservoir that have impact on history matching

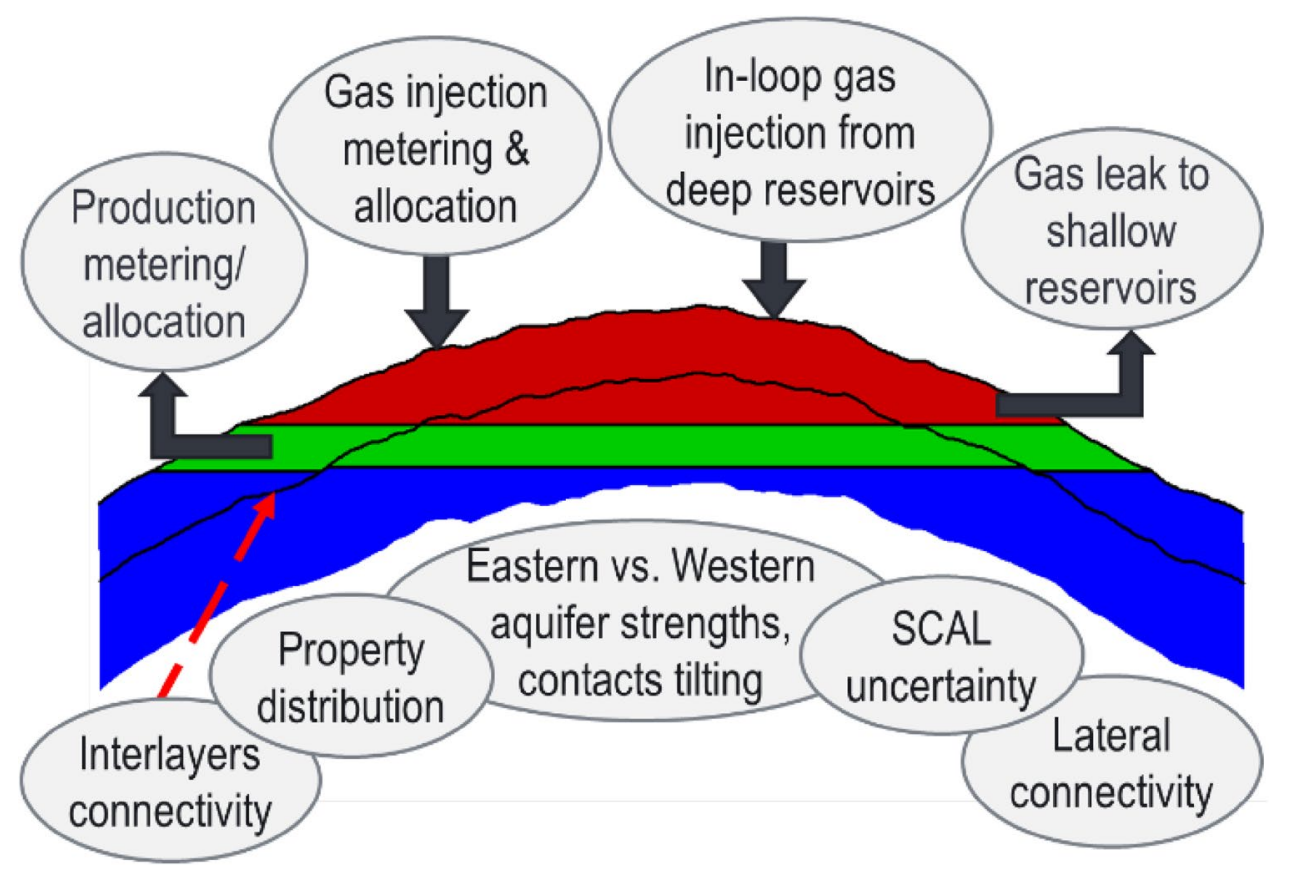

the calculated saturation from Saturation Height Function (SHF) vs. resistivity log-derived saturation.

Dynamic uncertainties included relative permeability, end points, $\mathrm{KV} / \mathrm{KH}$, and aquifer strength, etc. Uncertainty descriptions of relative permeability curves incorporated lab measurement errors, representativeness of core data, etc. The range of end points around base values is estimated based on its relationship to a Flow Zone Indicator (FZI).

Some uncertainty sources were excluded from the history matching process due to practical limitations to find an efficient parameterization, such as gas injection volumes, structural uncertainties, horizontal well landing depths, vertical well lateral position, etc. A sensitivity analysis was performed to evaluate the impact of these uncertainties on the history matching performance and most likely values were selected.

Table 1 summarizes key uncertainty parameters. Sensitivity runs showed that history matching parameters are less sensitive to residual oil saturation under water displacement (Sorw) or gas displacement (Sorg). Since the field development strategy involved gas and water injection these uncertainty parameters were kept active in the sensitivity parameter matrix to assess their impact on the recovery factor during the prediction phase.

\section{History matching}

The objective of the history matching phase was to calibrate the geological model and to generate multiple history matched scenarios that would address key uncertainties and hence assess their impact on the field development strategy.

A structured approach was followed to reach to an acceptable history match including the following items: Definition of objective functions that represent a weighted average mismatch between simulated and observed production and pressure data. The global objective function aggregated partial field and well related objectives functions according to their assigned weight and standard deviation as defined by Eq. 1 .

$Q(m)=\sum_{i} \omega_{i} \frac{\left(S_{i}(m)-O_{i}\right)^{2}}{\sigma_{i}^{2}}$,

where $S$ and $O$ describe simulated and observed measurement data, $m$ describes the set of model parameters, and $\sigma$ is the standard deviation of the measurement errors. $\omega$ is a weight factor which is used to prioritize data points.

Wells are ranked based on their cumulative oil production and data reliability. Weights were assigned to prioritize well contributions accordingly. Wells with a suspected incorrect production data due to measurements, back-allocation or well integrity issues (tubing leakage, behind casing channeling, etc.) are given a low weight or are excluded from the objective function. Documented measurement errors of historical production data are 
Table 1 XX-XY reservoir uncertainty matrix

\begin{tabular}{|c|c|c|c|}
\hline & Parameter & $\begin{array}{c}\text { Parameter } \\
\text { type }\end{array}$ & Comment \\
\hline & Structure & Categorical & $\begin{array}{l}\text { Uncertainty due to time depth, } \\
\text { conversion, wells, wells } \\
\text { seismic tie }\end{array}$ \\
\hline & $\begin{array}{c}\text { 3D } \\
\text { Connectivity } \\
\text { Factors }\end{array}$ & Continuous & $\begin{array}{l}\text { To account for the effects of } \\
\text { sub-seismic stratigraphic detail } \\
\text { not included in the model }\end{array}$ \\
\hline & $\begin{array}{l}\text { Stochastic } \\
\text { Shales }\end{array}$ & Continuous & $\begin{array}{l}\text { Random coverage of } \\
\text { shales/calcite barriers }\end{array}$ \\
\hline & Porosity & Continuous & $\begin{array}{l}\text { Measurement uncertainty at } \\
\text { well level }\end{array}$ \\
\hline & $\begin{array}{c}\text { Horizontal } \\
\text { Permeability }\end{array}$ & Continuous & $\begin{array}{l}\text { Permeability / Porosity } \\
\text { transform. FZI based function. } \\
\text { Calibration to PTA }\end{array}$ \\
\hline & $\mathrm{Kv} / \mathrm{Kh}$ & Continuous & $\begin{array}{l}\text { Vertical permeabillity. } \\
\text { Upscaling dependent }\end{array}$ \\
\hline & $\begin{array}{c}\text { Fluid } \\
\text { Contacts }\end{array}$ & Continuous & $\begin{array}{l}\text { Indepdent OGOC and OWOC } \\
\text { contacts }\end{array}$ \\
\hline & $\begin{array}{c}\text { Relative } \\
\text { Permeability }\end{array}$ & Continuous & $\begin{array}{l}\text { Cores correlations, } \\
\text { measumentsor upscaling } \\
\text { uncertainties }\end{array}$ \\
\hline & PVT & Continuous & $\begin{array}{l}\text { Based on fluid measurements } \\
\text { or correlations }\end{array}$ \\
\hline & $\begin{array}{l}\text { Mechanical } \\
\text { Skin }\end{array}$ & Continuous & Skin, productivity uncertainty \\
\hline & Injectivity & Categorical & Injectivity decline over time \\
\hline 1 & $\begin{array}{c}\text { Well count } I \\
\text { placement }\end{array}$ & Categorical & $\begin{array}{l}\text { Well count and placement } \\
\text { tuned to categorical static } \\
\text { model }\end{array}$ \\
\hline
\end{tabular}

captured by the standard deviation; for example, oil rate was assigned a standard deviation of $10 \%$ whilst GOR and water cut are assigned standard deviation of $40 \%$ and $20 \%$, respectively.

To identify parameters with a strong impact on the history matching quality, sensitivity, and screening runs were performed using Plackett -Burman experimental design. This step was performed to ensure the provided parameter combination and ranges would provide a solution to the history matching problem and to understand key variables. A revision of the uncertainty matrix/base case model was done to improve the local match, especially on key wells. This was followed by another sensitivity/screening phase using Latin Hypercube, which helped to thoroughly explore the solution space to establish trends/correlations to effectively tune the model in the next phase.

An extensive data base was formed and was analyzed using a proxy-based correlation technique for ranking parameter contributions, with a most likely impact on

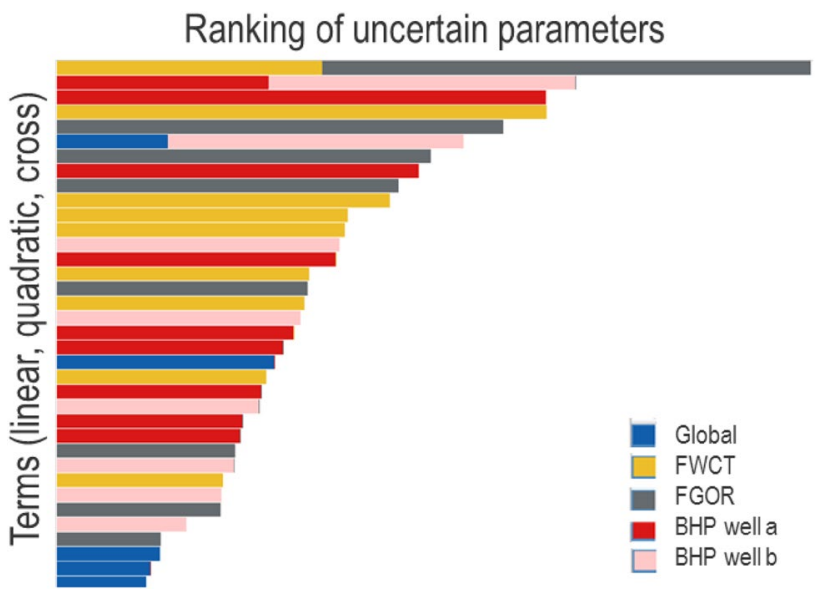

Fig. 10 Top key parameters with high impact on field responses and pressure match. Terms represent linear, quadratic and correlation terms

history matching responses on field and well level. Most important uncertainties were kept and less effective ones were disregarded.

Figure 10 shows the relative impact of parameter combinations from Latin Hypercube runs on global and partial mismatch response. The top parameters affecting the history match are gas oil and water oil contacts, vertical to horizontal permeability ratio, global permeability, oil relative permeability, and connectivity between XX and XY units.

Additional Latin Hypercube runs were performed to respect the reduced input parameter set. In every phase, the objective function was further tuned. Individual responses were revisited and only important history events were selected. Wells and responses with anti-correlation behavior were removed from the objective function definition.

The available simulation cases were ranked based on global and partial mismatches and a maximum threshold was applied for each parameter in order to eliminate poor history matched cases from being included in a training dataset for automatic proxy modeling during the MCMC phase. Figure 11 shows as stacked plot based on the global objective function and individual rates and pressure contribution.

Markov Chain Monte-Carlo (MCMC) including automatic proxy modeling technique was used to tune and sample acceptable history matching solutions.

8 history matched cases were selected out of a few hundred simulation cases. These candidates were moved to prediction. Although some of these cases did not match all objectives, they have been considered to ensure a reasonable estimate of recovery range, considering the uncertainty level attached to production data measurements and allocation. A comparison of the simulation results of these eight cases with historical production data is shown in Fig. 12. Among 


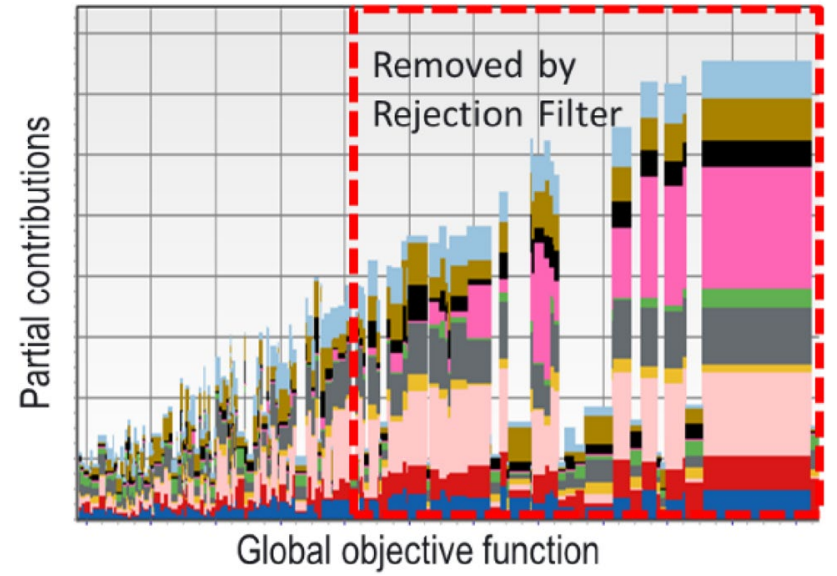

Fig. 11 Stacked plot showing partial objective contributions (individual rate and pressure mismatch values) against the global objective function value. The rejection filter is applied to remove cases with a poor history matching quality (red rectangle)

these cases a base case model was selected for further local tuning.

\section{Forecasting under uncertainty}

The history matching workflow was designed to deliver a selection of history matched models which are used for estimating prediction uncertainty. 8 history matched cases were selected to test the field development plan outcomes. A range of oil rate forecast $\&$ recoveries are generated as shown in Fig. 13. The divergence of field performance predictions represents the risk assessment based on the subsurface uncertainty description.
3D oil saturation results from the selected cases are used to generate probability maps of mobile oil at different time steps, to reveal probabilistic estimates of its spatial distribution.

The multi-realization ensemble of history matched cases allows us to ask: What is the probability to find an oil saturation in an area of interest which is above a certain threshold value? This allows to identify areas with bypassed hydrocarbons which may not be accessible or depleted in the current field production scenario given all considered uncertainties.

Figure 13 shows the probability map of oil saturation being above a desired threshold of oil saturation of the field development target reservoir, calculated on grid cell level including results from all eight simulation cases.

By overlaying the original infill wells trajectories on mobile oil saturation probability map as shown in Fig. 14 (upper), it becomes clear that the three wells are positioned in low-probability areas. As a result, positions of all three wells were optimized to target high-probability spots as shown in Fig. 14 (lower). Simulation runs for a set of history matched reservoir models (Fig. 13) on optimized infill well positions (Fig.14) confirmed an average incremental increase of the recovery factor (Fig. 15) by $3 \%$.

\section{Estimating recovery factor ranges}

From eight history matched simulation models including a forecast scenario, an approximate distribution for field oil initially in place, ultimate recovery, and recovery factor was created. The recovery factor was calculated as a fraction of ultimate oil recovery over in oil place volume. The dataset of eight prediction runs, shown in Fig. 15 are used to define normal distribution functions by matching approximate P10 and P90 values. A Monte Carlo sampling
Fig. 12 Shows field oil rate, water cut, pressure, and GOR simulated vs. observed data for the selected cases
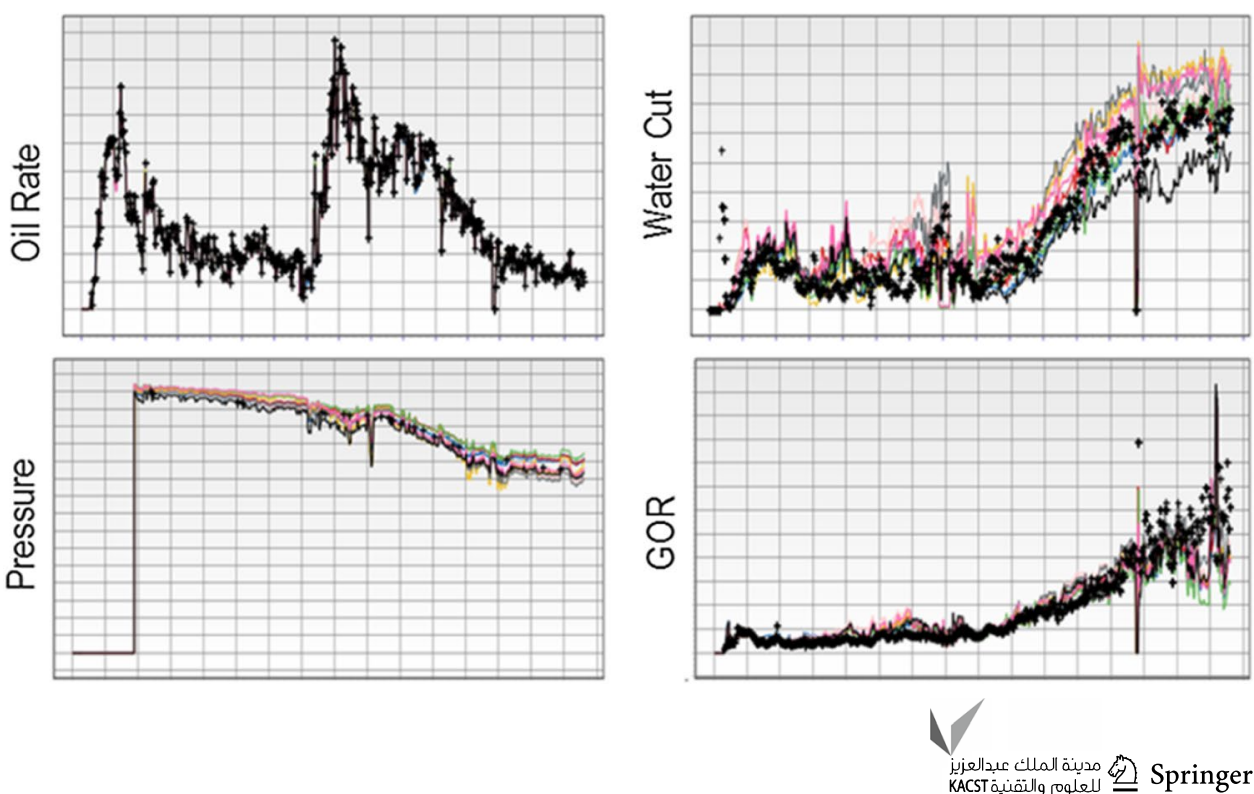
Fig. 13 Maps on the top show the probability distribution for saturation oil $P\left(S_{\text {oil }}\right) \geq 0.4$ at the start of production (left), end of history (mid), and at the end of the forecast period (right). Profiles below show field oil production rate and total for all history matched solution cases as well as ultimate recovery for the field development plan

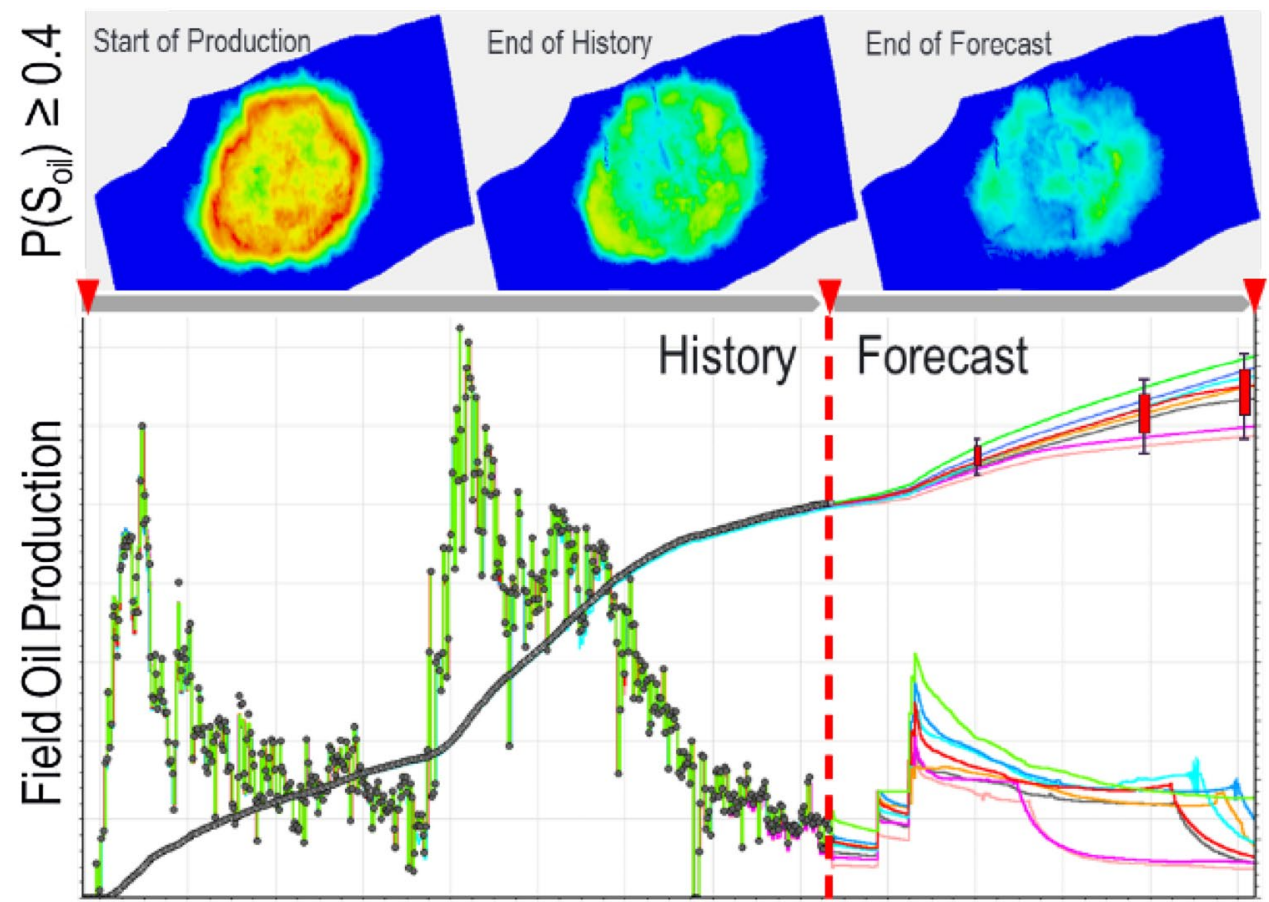

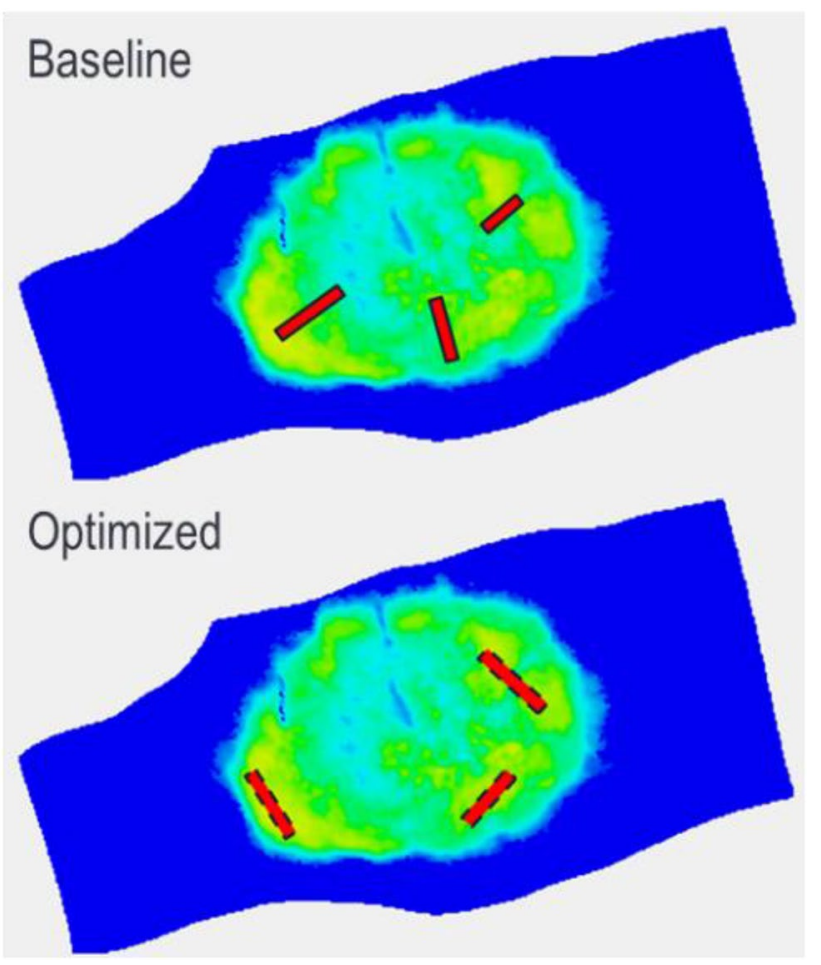

Fig. 14 Infill well locations (red bars) shown on mobile oil probability map at the end of history, baseline FDP design targets (upper), optimized targets (lower) process fills in data to generate a smooth distribution function for recovery factor shown in the right plot of Fig. 15.

All simulation runs used for prediction are fully validated in terms of history matching error criteria and consistency with the initial geological model. The uncertainty distribution in Fig. 15 (right) makes a strong assumption on the uncertainty spread in the simulated data set shown in Fig. 15 (left). Distribution mean and variance may change once additional history matched simulation models are added. However, it is expected that the range remains stable based on the given uncertainty matrix definition in Table 1.

\section{Field development plan and optimization under uncertainty}

Under the discussed assumptions, the estimated recovery factor for the base development plan ranges within the P10 and P90 level from 32 to $38 \%$. This prepares the conceptual basis of a risk assessment of any new field development plan under subsurface uncertainties.

Optimized field development strategies need to be verified against the ensemble of history matched reservoir simulation models to investigate risk. Optimization workflows ideally include multiple representative reservoir models to verify robustness and optimization potential of any optimized field development scenario under uncertainty. 
Fig. 15 Left: 8 selected prediction cases shown in a cross plot ultimate recovery vs. field oil initially in place. The color coding of the data points indicates the range of recovery factors. Right: a distribution of recovery factors is created from Monte Carlo Simulation with P10, P50 and P90 estimates

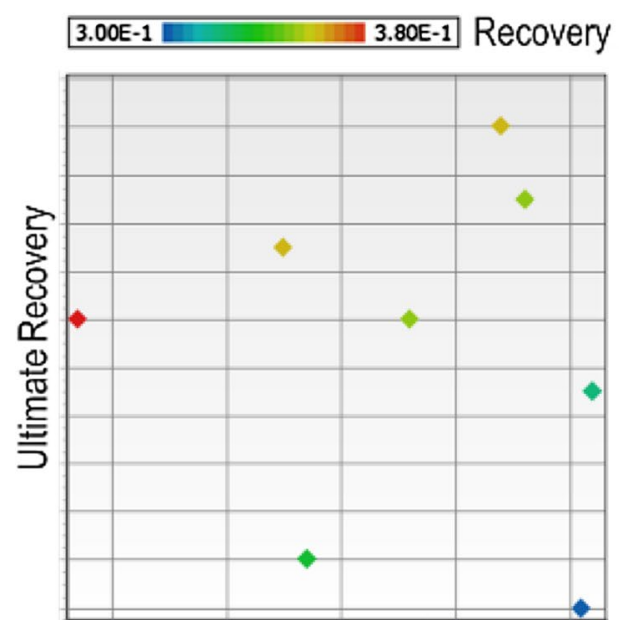

Field Oil Initially in Place

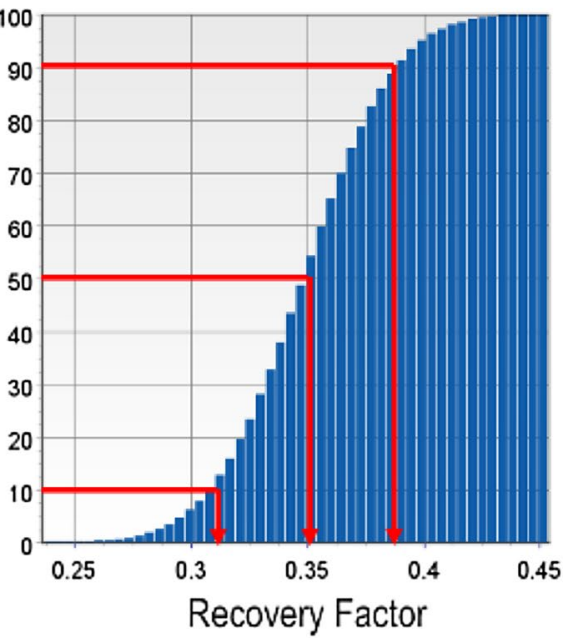

\section{Lesson learned}

This section summarizes key experiences and recommendations:

- Include and keep important uncertainty parameters that have minor impact in the history matching phase but may impact future field performance.

- The objective function should capture only major events such as production trends, breakthrough time and should avoid well data with erroneous measurement.

- The global objective function must be a good indicator for an acceptable history match. This requires a tuning process which should not be ignored. Balance partial field and well objective contributions and identify objective contributions which show competing performance behavior. Depending on project objectives, it is recommended to remove those responses and to focus on overall field and/or regional responses.

- Launch sensitivity studies to understand key parameter contributions that will impact the history matching quality at both field and well level prior launching history matching processes.

- Limit the number of uncertainty parameters in every history matching evaluation phase. Results must be possible to analyze, and conclusions must be drawn. The number of required simulation runs should be ideally completed within a working day.

- History match on reservoir level is not necessarily correlated with a good match on well level. It is recommended to give more emphasis on reservoir level matching.

- Use of probability maps based on candidate solutions sampled from the posterior distribution helps to identify areas of high risk and effectively optimize infill location.

\section{Summary and conclusion}

In this work, we discuss a practical risk assessment of a field development plan of a complex oil rim reservoir under subsurface uncertainties.

- A robust and comprehensive understanding was established on key field uncertainties before embarking on history matching exercise. This involved a series of discussion and interaction among the team members to form a fit for purpose uncertainty matrix.

- Static and dynamic reservoir models were calibrated based on historical production data and key uncertainties were addressed.

- Probabilistic modeling helped to capture key uncertainties for generating a range of forecast cases and expected recovery ranges for better decision making.

- Use of probability maps helped to de-risk and optimize the planned infill well locations. The result of the optimization work is additional $3 \%$ of recovery factor in average.

In conclusion, a set of a calibrated models were generated to support key reservoir management decisions and to guide further field development evaluations. A robust effective workflow was established to deliver a value-driven risk assessment that can be replicated in the industry.

Acknowledgements The authors of this paper would like to thank the management of PETRONAS Sarawak Oil Resource and Schlumberger for permitting this work to be published. Conclusions and opinions stated in this paper are those of the authors and do not necessarily represent those of PETRONAS Sarawak Oil Resource.

Open Access This article is distributed under the terms of the Creative Commons Attribution 4.0 International License (http://creativeco 
mmons.org/licenses/by/4.0/), which permits unrestricted use, distribution, and reproduction in any medium, provided you give appropriate credit to the original author(s) and the source, provide a link to the Creative Commons license, and indicate if changes were made.

\section{References}

Bhark EW, Dehghani K (2014) Assisted history matching benchmarking: design of experiments-based techniques. Soc Petrol Eng. https ://doi.org/10.2118/170690-MS

Chugunov NR (2015) Method for adaptive optimization of EOR performance under uncertainty. Soc Petrol Eng. https://doi. org/10.2118/173295-MS

Chugunov $\mathrm{N}$ et al (2013) Global sensitivity analysis for crosswell seismic and nuclear measurements in $\mathrm{CO}_{2}$ storage projects. Geophysics 78(3):WB77-WB87. https://doi.org/10.1190/geo2012-0359.1

Hanea R, Evensen G, Hustoft L, Ek T, Chitu A, Wilschut F (2015) Reservoir management under geological uncertainty using fast model update. Soc Petrol Eng. https://doi.org/10.2118/173305-MS

Komin MM-R (2018) Multivariate asset assessment: fast and reasonable decision on oil rims development on the example of a unique field in the arctic onshore. Soc Petrol Eng. https://doi. org/10.2118/191612-18RPTC-MS
Nandurdikar NS, Wallace L (2011) Failure to produce: an investigation of deficiencies in production attainment. Soc Petrol Eng. https:// doi.org/10.2118/145437-MS

Schulze-Riegert R, Nwakile M, Skripkin S, Willen Y (2016) Scalability and performance efficiency of history matching workflows using MCMC and adjoint techniques applied to the norne north sea reservoir case study. Soc Petrol Eng. https://doi.org/10.2118/18010 6-MS

Schulze-Riegert R, Daniali A, Nwakile M, Selberg S, Skripkin S, Chugunov N, Carter J (2017a) Data analysis used in multiple-realization workflows for history matching — a north sea case study. Soc Petrol Eng. https://doi.org/10.2118/185877-MS

Schulze-Riegert R, Magdeyev I, Komin M, Chernayak V (2017b) Brownfield development optimization under uncertainty-a structured workflow design for complex case scenarios. Soc Petrol Eng. https://doi.org/10.2118/187856-MS

Slotte PA, Smorgrav E (2008) Response surface methodology approach for history matching and uncertainty assessment of reservoir simulation models. Soc Petrol Eng. https://doi.org/10.2118/113390-MS

Publisher's Note Springer Nature remains neutral with regard to jurisdictional claims in published maps and institutional affiliations. 